\title{
Predicate Logic and Tree Automata with Tests
}

\author{
Ralf Treinen* \\ Laboratoire de Recherche en Informatique \\ Université Paris-Sud \\ F-91495 Orsay cedex, France \\ http://www.lri.fr/ treinen
}

\begin{abstract}
We investigate the question whether the well-known correspondence between tree automata and the weak second order logic of two successor functions (WS2S) can be extended to tree automata with tests. Our first insight is that there is no generalization of tree automata with tests that has a decidable emptiness problem and that is equivalent to the full class of formulae in some extension of WS2S, at least not when we are asking for an conservative extension of the classical correspondence between $W S 2 S$ and tree automata to tree automata with tests.

As a consequence we can extend the correspondence between tree automata and WS2S to automata with tests only when we admit a restriction of the class of formulae. We present a logic, called WS2Sy, and a restriction of the class of formula, called uniform, that is equivalent to tree automata with tests.
\end{abstract}

\section{Introduction}

The equivalence of tree automata and weak second-order logic of 2 successor functions, short WS2S, is known for more then 30 years [10]. During the current decade, a lot of work has been done on classes of tree automata that are stronger than classical tree automata (see [4] and [8] for a survey). The general frame to obtain these stronger classes of tree automata is to augment the automaton model with tests. In the case of tree automata with tests between brothers [1], for instance, we can write a transition rule like $f\left(q_{1}, q_{2}\right) \rightarrow^{1=2} q$ that will accept a tree $f\left(t_{1}, t_{2}\right)$ in state $q$ if $t_{1}$ is accepted in state $q_{1}, t_{2}$ is accepted in $q_{2}$, and if in addition $t_{1}=t_{2}$. These tests increase in a considerable way the expressiveness of tree automata. Tree automata with equality tests between brothers as sketched above can for instance recognize the set of balanced trees (which is not possible with classical tree automata).

Defining new classes of tree automata is of course only interesting when the usual "nice" properties of automata are preserved. The most important of these is the decidability of the emptiness of the language recognized by such an automaton. Furthermore we expect a good class of recognizable languages to

^ Partially supported by the Esprit Working Group 22457 - CCL II

J. Tiuryn (Ed.): FOSSACS 2000, LNCS 1784, pp. 329 343 2000.

(C) Springer-Verlag Berlin Heidelberg 2000 
be closed under operations such as union, intersection, complement and (maybe restricted classes of) homomorphisms and inverse homomorphisms.

Several classes of tree automata with "nice" properties have been identified (see [4] for an excellent overview of tree automata and tree automata with tests). In light of the above mentioned equivalence between tree automata and the logic $W S 2 S$ it is a natural question whether there are similar logical characterizations of tree automata with tests. Before attacking this question we will try to explain in an informal manner the logic $W S 2 S$ and its correspondence with tree automata.

The second-order logic WS2S comes with a standard interpretation. There are two sorts: the sort of words over the alphabet $\{1,2\}$ and the sort of finite sets of words. There are a constant for the empty word, unary functions to append a symbol 1, resp. 2 at the end of a word, and the elementship relation between words and sets. An example of a formula in this logic, expressing that the word $x$ is a prefix of the word $y$ is (the convention is that word variables are written in lower case and set variables in upper case):

$$
\forall Y(y \in Y \wedge \forall z([z 1 \in Y \vee z 2 \in Y] \rightarrow z \in Y) \rightarrow x \in Y)
$$

A tree automaton with $k$ states can be translated into a formula of WS2S in the following sense: We represent a tree over an alphabet of $n$ symbols by $n$ sets, each of them representing the occurrences (addresses of nodes) of the tree marked with one the symbols. The formula has to state that these sets are pairwise disjoint (since an occurrence can carry only one symbol), that their union is closed under prefix (using the prefix predicate defined above) and that the arity of the function symbols is respected, that there is an assignment of states to the occurrences (this is expressed by an existential quantification over $k$ sets similar to what we did to represent the term itself) respecting the transition rules of the automaton such that the root is marked with an accepting state.

Given a formula $\phi$ with $n$ free variables (by a slight variation of the logic we can assume that only set variables are involved) we first have to say how to represent an $n$-tuple of sets as a tree: We take as tree signature the $n$-tuples over $\{0,1\}$, a tree representing a $n$-tuple of sets has at occurrence $\pi$ the symbol 1 in the $i$-th component if and only if $\pi$ belongs to the $i$-th set. Since all sets in WS2S are finite a finite tree is sufficient (there is also a technically much more involved variant for infinite sets and infinite trees due to Rabin [7]). Now, the idea to get an automaton that corresponds to a given formula is to first construct automata for the atomic formula of the logic, and then to apply the closure of tree automata by union, complement and projection (corresponding to disjunction, negation and existential quantification).

In fact there is a problem with the translation of formulae into automata that is easily overseen when sketched as we did above: For this translation to work we need an additional closure property of tree automata (which, luckily, does hold for classical tree automata): closure by cylindrification. The problem is apparent when we have constructed a tree automaton for a formula $\phi\left(X_{1}, X_{2}\right)$ and another one for a formula $\psi\left(X_{2}, X_{3}\right)$ and when we now want to construct from these the 
automaton for $\phi\left(X_{1}, X_{2}\right) \wedge \psi\left(X_{2}, X_{3}\right)$. We cannot just take the intersection of these two automata since the two "components" of the trees recognized by the respective automata do not correspond to the same variables. In fact we have to cylindrify both automata, that is add to the first automaton a third component and to the second automaton a new first component that are in fact completely ignored by the rules of the automata (the origin of the name "cylindrification" is the intuition of a two-dimensional geometrical figure that is slided along the third dimension through space). Finally we can take the intersection of the two cylindrified automata.

Here lies the problem when we ask for a logical characterization of tree automata with tests: The closure properties of the logic, including the "implicit" closure by cylindrification that simply stems from the presence of variables in the logic, require any class of automata that corresponds to the full class of formulae of some logic to be closed under cylindrification.

If we need cylindrification for our class of automata to correspond to some logic then we could simply extend our automaton model accordingly and try to show that the "nice" properties of automata still hold. The automata model that we get from Tree Automata with Tests (TAT) when we close under cylindrification are Tree Automata with Component-wise Tests (TACT) that will be formally defined in Section 3. Unfortunately, emptiness of TACT-automata is undecidable as we will show in Section 4, that is, our original program simply has to fail.

Hence we propose to ask for a subset of the set of formulae of some suitable logic that is equivalent to our class of formulae. We define in Section 5 such a logic WS2Sy and its two subclasses of so-called restricted and uniform formulae. We state the exact correspondence between TACT and TAT automata and the subclasses of restricted, resp. uniform formulae of WS2Sy in Section 6 and prove this correspondence in Section 7 and 8 The technical part of the paper only considers automata with tests between brothers, we briefly discuss extension to other classes of automata with tests in Section 9,

\section{Preliminaries}

A signature $\Sigma$ consists of a set of symbols (also denoted $\Sigma$ ) and an arity function $\alpha: \Sigma \rightarrow \mathbf{N}_{0}$. Often one writes $\Sigma_{n}$ for $\{f \in \Sigma \mid \alpha(f)=n\}$. For a signature $\Sigma$ the set $T(\Sigma)$ of $\Sigma$ ground terms is the smallest set such that if $f \in \Sigma_{n}$ and $t_{1}, \ldots, t_{n} \in T(\Sigma)$ then $f\left(t_{1}, \ldots, t_{n}\right) \in T(\Sigma)$. The set of occurrences $O(t)$ of a tree $t=f\left(t_{1}, \ldots, t_{n}\right)$ is $\{\epsilon\} \cup \bigcup_{i=1}^{n}\left\{i w \mid w \in O\left(t_{i}\right)\right\}$. If $\pi \in O(t)$ then the subtree $\left.t\right|_{\pi}$ of $t$ at $\pi$ is defined by $\left.t\right|_{\epsilon}=t$ and $\left.f\left(t_{1}, \ldots, t_{n}\right)\right|_{i \pi}=\left.t_{i}\right|_{\pi}$.

Given signatures $\Sigma, \Gamma$, a renaming $\rho$ is a function $\rho: \Sigma \rightarrow \Gamma$ such that $\alpha(\rho(f)) \leq \alpha(f)$ for all $f \in \Sigma$. A renaming $\rho: \Sigma \rightarrow \Gamma$ extends to a tree homomorphism $\rho: T(\Sigma) \rightarrow T(\Gamma)$ by $\rho\left(f\left(t_{1}, \ldots, t_{n}\right)\right)=(\rho(f))\left(\rho\left(t_{1}\right), \ldots, \rho\left(t_{\alpha(\rho(f))}\right)\right)$ (hence, arguments not needed in $\Gamma$ are simply dropped).

An instance of the Post Correspondence Problem (short: PCP) is a finite sequence $P=\left(\left(p_{i}, q_{i}\right)\right)_{i=1, \ldots, m}$ of pairs of words from $\{a, b\}^{*}$. A solution of such 
an instance of PCP is a nonempty sequence $\left(i_{1}, \ldots, i_{n}\right), 1 \leq i_{j} \leq m$, such that $p_{i_{1}} \cdots p_{i_{n}}=q_{i_{1}} \cdots q_{i_{n}}$. According to a classical result due to Post [6], it is undecidable whether an instance of the PCP has a solution or not.

\section{Tree Automata with Component-wise Tests}

Definition 1 (Tuple Signature). For any finite alphabet $\Gamma$ and $n \geq 0$, the signature $\Gamma^{(n)}$ consists of the set of function symbols $(\Gamma \cup\{\perp\})^{n}$, where $\perp$ is a new symbol. The symbol $(\perp \ldots \perp)$ is a constant, all other symbols are binary.

Example 1. Let $\Gamma=\{0,1\}$. The signature $\Gamma^{(2)}$ contains the binary symbols $00,01,0 \perp, 10,11,1 \perp, \perp 0, \perp 1$ and the constant $\perp \perp$.

Definition 2 (Projection and Component of a Tree). Let $\tau$ be a $\Gamma^{(n)}$-tree and $1 \leq i \leq n$.

1. The $i$-th component of $\tau$, denoted $\tau^{i}$, is the $\Gamma^{(1)}$-tree given by the renaming $\left(f_{1} \ldots f_{i} \ldots f_{n}\right) \mapsto f_{i}$ for all $f_{j} \in \Gamma \cup\{\perp\}$.

2. The $i$-th projection of $\tau$, denoted $\tau^{\langle i\rangle}$, is the $\Gamma^{(n-1)}$-tree given by the renaming $\left(f_{1} \ldots f_{i-1} f_{i} f_{i+1} \ldots f_{n}\right) \mapsto\left(f_{1} \ldots f_{i-1} f_{i+1} \ldots f_{n}\right)$ for all $f_{j} \in \Gamma \cup\{\perp\}$.

Note that both projection and selection of a component can change the set of occurrences of a tree since a binary symbol may become a constant, as is illustrated in the example given on Figure 1.

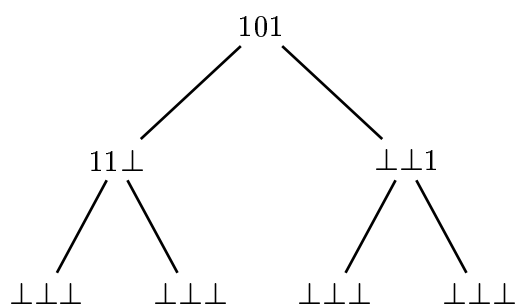

(a) Tree $\tau$

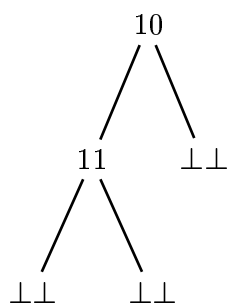

(b) $\tau^{\langle 3\rangle}$

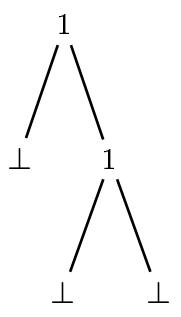

(c) $\tau^{3}$

Fig. 1. Example of projection and component.

Definition 3 (Tree Automata). $A$ tree automaton with component-wise tests between brothers (short: TACT) is a quintuplet $\left(\Gamma, n, Q, Q_{f}, \Delta\right)$ where

$-\Gamma$ is a finite alphabet and $n \geq 1$,

$-Q$ is a finite set of states, 
$-Q_{f} \subseteq Q$ is the set of accepting states,

$-\Delta$ is a set of transition rules of one of the following forms:

- $(\perp \ldots \perp) \rightarrow q, q \in Q$.

- $f\left(q_{1}, q_{2}\right) \rightarrow^{c} q$ where $c$ is a boolean combination of tests $1 . i=2 . i$ with $1 \leq i \leq n, f \in \Gamma^{(n)}-(\perp \ldots \perp), q, q_{1}, q_{2} \in Q$.

$A$ tree automaton with tests between brothers (short: TAT) is a TACT automaton $\left(\Gamma, n, Q, Q_{f}, \Delta\right)$ where all tests of rules in $\Delta$ are either True, or $1.1=$ $2.1 \wedge \ldots \wedge 1 . n=2 . n$, or $1.1 \neq 2.1 \vee \ldots \vee 1 . n \neq 2 . n$.

$A$ tree automaton (short: TA) is a $\operatorname{TACT}\left(\Gamma, n, Q, Q_{f}, \Delta\right)$ where all tests of rules in $\Delta$ are True.

Note that in a TAT all tests operate uniformly on all components. Hence TAT's are exactly the tree automata with tests between brothers introduced in [1, and TA are classical tree automata. In the examples we will simply drop a test True on a transition rule and write it as the rule of a classical tree automaton.

Definition 4. The TACT A tests only on the set of components $I$ if whenever $A$ contains a transition rule with a test $c$ and $1 . i=2 . i$, resp. $1 . i \neq 2 . i$ is an atomic test in $c$, then $i \in I$.

Definition 5 (Acceptance by a TACT). A tree $t$ satisfies the test $1 . i=2 . i$ iff $\left(\left.t\right|_{1}\right)^{i}=\left(\left.t\right|_{2}\right)^{i}$, this extends in the canonical way to boolean combinations of tests.

The rewriting relation of a TACT $A=\left(\Gamma, n, Q, Q_{f}, \Delta\right)$ is the smallest binary relation $\rightarrow{ }_{A} \subseteq T\left(\Gamma^{(n)}\right) \times Q$ with

$-a \rightarrow_{A} q$ if $a \rightarrow q \in \Delta$

$-f\left(t_{1}, t_{2}\right) \rightarrow_{A} q$ if $f\left(q_{1}, q_{2}\right) \rightarrow^{c} q \in \Delta, f\left(t_{1}, t_{2}\right)$ satisfies $c, t_{1} \rightarrow_{A} q_{1}$ and $t_{2} \rightarrow q_{2}$.

The automaton $A$ accepts a tree $t$ if $t \rightarrow_{A} q$ for some accepting state $q \in Q_{f}$. The set $L_{A}$ recognized by $A$ is the set of all trees accepted by $A$.

Definition 6 (Deterministic and Complete TACT). A TACT A is deterministic if for every tree $t$ there is at most one state $q$ such that $t \rightarrow_{A} q$, and complete if for every tree $t$ there is at least one state $q$ with $t \rightarrow_{A} q$.

The following proposition is immediate from our definitions:

Proposition 1. Every TA-recognizable set is TAT-recognizable, and every TATrecognizable set is TACT-recognizable. 


\section{The Emptiness Problem for TACT}

Theorem 1. The emptiness-problem for TACT automata (even when restricted to the class of TACT without negative constraints) is undecidable.

Proof: We reduce the Post Correspondence Problem to the emptiness problem of TACT automata. Let $P=\left(\left(p_{i}, q_{i}\right)\right)_{i=1, \ldots, m}$ be an instance of PCP.

First some terminology: a tree $t \in T\left(\Gamma^{(n)}\right)$ is a comb if every binary node of $t$ has the constant $(\perp \ldots \perp)$ as its left son. For example, the tree of Figure 2 is not a comb but its right subtree is. For a comb $t \in T\left(\Gamma^{(n)}\right)$ and $1 \leq i \leq n$, the $i$-th word coded by $t$ is the word $t \downarrow_{i} \in \Gamma^{*}$ defined by

$$
t \downarrow_{i}= \begin{cases}\epsilon & \text { if } t^{i}=\perp \\ \left.c \cdot t\right|_{2} \downarrow_{i} & \text { if } t^{i}=c\left(\perp,\left.t\right|_{2}\right)\end{cases}
$$

For instance, if $t$ is the right subtree of the tree depicted in Figure 2 then $t \downarrow_{1}=$ $a a b b$ and $t \downarrow_{3}=f a$.

We define the signature of our automaton as $\{f, a, b\}^{(4)}$. The automaton $L_{P}$ is constructed in three steps:

The first step is to construct an automaton that accepts a comb if it encodes two pairs of words such that the second pair is obtained from the first pair by application of one step of the PCP instance P. For reasons that will be apparent in the last step this automaton comes in a positive and in a negative version that differ in the choice of the components that code the two pairs. More exactly:

For any word $v \in \Gamma^{*}$, let $A_{v}^{i, j}$ for $i \neq j$ be the automaton that accepts $t$ iff $t$ is a comb, $t \downarrow_{i}=f w$ and $t \downarrow_{j}=w v$ for some $w \in \Gamma^{*}$. If $v=v_{1} \cdots v_{n}$ then the rules of the automaton are (here for $i=1$ and $j=2$ ), where _ stands for any symbol from $\{a, b, f, \perp\}$ and $\alpha, \beta$ for any symbol from $\{a, b\}$ :

$$
\begin{aligned}
(\perp \perp \perp \perp) & \rightarrow q_{l} \\
(\perp \perp \perp \perp) & \rightarrow q_{n} \\
(\perp \perp--)\left(q_{l}, q_{n}\right) & \rightarrow q_{n} \\
\left(\perp v_{i--}\right)\left(q_{l}, q_{i}\right) & \rightarrow q_{i-1} \quad i=2, \ldots, n \\
\left(f v_{1--}\right)\left(q_{l}, q_{1}\right) & \rightarrow q_{0} \\
\left(\alpha v_{1--}\right)\left(q_{l}, q_{1}\right) & \rightarrow q_{\alpha} \\
\left(\alpha \beta_{--}\right)\left(q_{l}, q_{\beta}\right) & \rightarrow q_{\alpha} \\
\left(f \beta_{--}\right)\left(q_{l}, q_{\beta}\right) & \rightarrow q_{0}
\end{aligned}
$$

The only accepting state is $q_{0}$.

From the boolean closure of (classical) tree automata we get an automaton $A_{P}^{+}$that accepts $t$ iff $t$ is a comb and for some $i$ we have that $t \downarrow_{1}=f w_{1}$, $t \downarrow_{2}=f w_{2}, t \downarrow_{3}=w_{1} p_{i}$ and $t \downarrow_{4}=w_{2} q_{i}$. Analogously, the automaton $A_{P}^{-}$accepts $t$ iff $t$ is a comb and for some $i$ we have that $t \downarrow_{1}=w_{1} p_{i}, t \downarrow_{2}=w_{2} q_{i}, t \downarrow_{3}=f w_{1}$ and $t \downarrow_{4}=f w_{2}$. Let $Q^{+}$resp. $Q^{-}$be the accepting states of these two automata. In case of the tree $t$ of Figure [2, for example, $A_{P}^{+}$accepts $\left.t\right|_{12}$ and $A_{P}^{-}$accepts $\left.t\right|_{2}$. 
The second step is to construct automata that in addition test whether the newly constructed pair is of the form $(w, w)$ for some non-empty word $w$. We can easily derive from $A_{P}^{+}$this automaton $A E_{P}^{+}$(with set of accepting states $Q_{=}^{+}$) that emulates $A_{P}^{+}$and verifies in addition that $t \downarrow_{3}=t \downarrow_{4} \neq \epsilon$, and analogously $A E_{P}^{-}$(with set of accepting states $Q_{=}^{-}$) that emulates $A_{P}^{-}$and verifies in addition that $t \downarrow_{1}=t \downarrow_{2} \neq \epsilon$. In case of the tree $t$ of Figure $2 A E_{P}^{-}$accepts $\left.t\right|_{2}$.

Note that the first two steps used only classical tree automata. The third step is now to build the final automaton which uses these four automata as sub-automata (we hence assume their states-spaces to be disjoint) to accept a solution sequence of $P$. This is where equality tests come in.

$$
\begin{array}{ccc}
(\perp \perp \perp \perp) & \rightarrow & p_{\perp} \\
(f f f f)\left(p_{\perp}, p_{\perp}\right) & \rightarrow & p^{+} \\
(f f f f)\left(p^{+}, q^{+}\right) & \rightarrow{ }^{1.1=2.1 \wedge 1.2=2.2} p^{-} & q^{+} \in Q^{+} \\
(f f f f)\left(p^{-}, q^{-}\right) \rightarrow^{1.3=2.3 \wedge 1.4=2.4} p^{+} & q^{-} \in Q^{-} \\
(f f f f)\left(p^{+}, q^{+}\right) \rightarrow^{1.1=2.1 \wedge 1.2=2.2} p_{f} & q^{+} \in Q_{=}^{+} \\
(f f f f)\left(p^{-}, q^{-}\right) \rightarrow^{1.3=2.3 \wedge 1.4=2.4} p_{f} & q^{-} \in Q_{=}^{-}
\end{array}
$$

where $p_{f}$ is the only accepting state. An example of the representation of the solution of an instance of PCP as accepted by this automaton is given in Figure2. In this example, the accepting run assigns the occurrence 11 the state $p^{+}$, the ocurrence 1 the state $p^{-}$and the occurrence $\epsilon$ the accepting state $p_{f}$.

The undecidability result holds even in case of signatures with only two components. For a proof, it suffices to encode in the above proof the first and second, respectively third and forth component of the signature into one symbol.

\section{The Logic WS2Sy}

The logic WS2Sy is a two-sorted logic with the sorts w (for word) and s (for set). We will write variables of sort $\mathrm{w}$ in lower case and variables of sort $\mathrm{s}$ in upper case. Function symbols are a constant $\epsilon$ of sort $\mathrm{w}$ and two monadic function symbols 1 and 2 of profile $\mathrm{w} \rightarrow \mathrm{w}$. We write applications of these function symbols in postfix notation, that is $x 1121$ for $1(2(1(1(x))))$. Relation symbols are $\in$ of profile $\mathrm{w} \times \mathbf{s}$, and for every $n \geq 1$ a $n+1$-ary predicate $S y$ of profile $\mathrm{w} \times \mathbf{s} \times \ldots \times \mathbf{s}$.

The standard interpretation of WS2Sy assigns the sort $\mathrm{w}$ as universe the set $\{1,2\}^{*}$ and the sort $\mathrm{s}$ as universe the set of all finite subsets of $\{1,2\}^{*}$. The constant $\epsilon$ is interpreted as the empty word, and the function 1 (resp. 2) as the function that appends 1 (resp. 2) to a word. The predicate symbol $\in$ denotes set membership. The synchronisation predicate $S y\left(w, M_{1}, \ldots, M_{n}\right)$ holds if for all $1 \leq i \leq n$ and all $x \in\{1,2\}^{*}$ we have that $w 1 x \in M_{i}$ iff $w 2 x \in M_{i}$.

The notion of a free occurrence of a variable in a formula is defined as usual. A WS2Sy-formula $\phi$ is called restricted if whenever $\phi$ contains a subformula of the form $S y\left(x, X_{1}, \ldots, X_{n}\right)$ then $X_{1}, \ldots, X_{n}$ are free variable occurrences (here, 


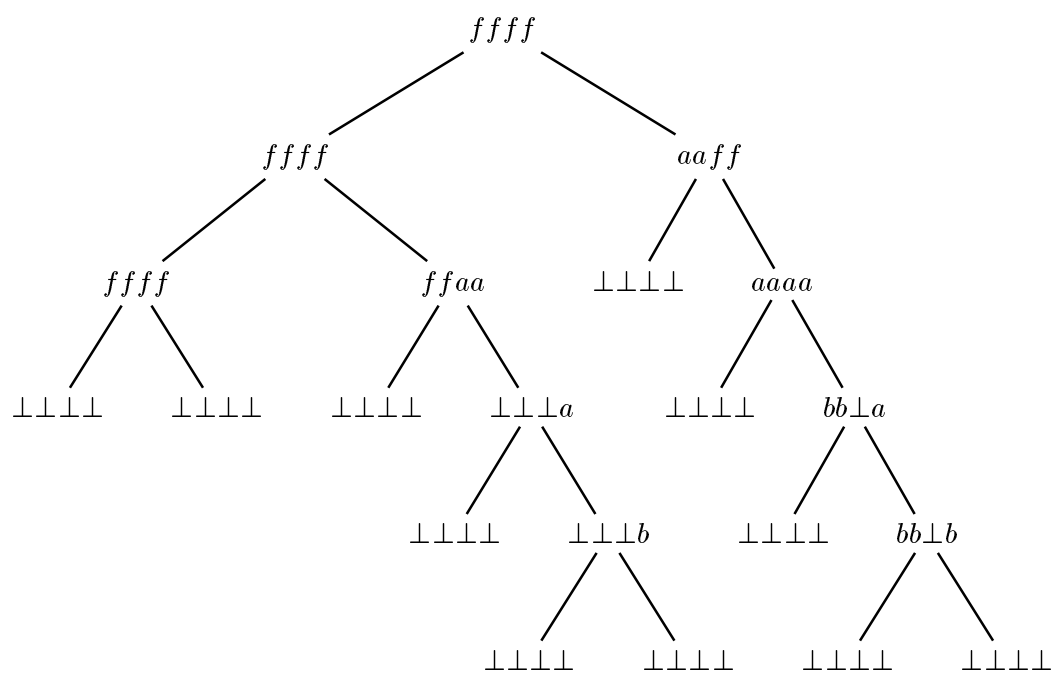

Fig. 2. Example of the representation of the solution $((\epsilon, \epsilon),(a, a a b),(a a b b, a a b b))$ of the PCP instance $((a, a a b),(a b b, b))$.

there is no restriction on $x$ being free or bound). A $W S 2 S y$-formula $\phi$ is called uniform if whenever $\phi$ contains a subformula of the form $S y\left(x, X_{1}, \ldots, X_{n}\right)$ then $X_{1}, \ldots, X_{n}$ are exactly the free variable occurrences of $\phi$ (hence, $x$ is bound). As a consequence, every uniform formula of WS2Sy is restricted, and the classical logic WS2S is obtained from WS2Sy by omiting the synchronisation predicates.

The logic WS2Sy allows to define the usual set-theoretic notions as union, intersection, singleton sets, etc. that we will use freely in the sequel. Furthermore, the prefix relation on words (denoted $x \leq y$ ) is expressible in WS2S as has been demonstrated in Section 1, hence in WS2Sy.

Following the classical translation of the logic WS2S into tree automata, we define the one-sorted version $W S 2 S y_{0}$ of $W S 2 S y$ where only set-variables and predicates on sets are used. The predicates of $W S 2 S y_{0}$ are $X_{1}=X_{2} 1, X_{1}=X_{2} 2$, $X_{1}=\epsilon, X_{1} \subseteq X_{2}$, and $S y\left(X_{0}, X_{1}, \ldots, X_{n}\right)$. In the standard interpretation of $W S 2 S y_{0}$ we have that $M_{1}=M_{2} 1$ is true iff $M_{2}$ is some singleton set $\left\{m_{2}\right\}$ and $M_{1}=\left\{m_{2} 1\right\}$ and analogous for $M_{1}=M_{2} 2 ; M_{1}=\epsilon$ is true iff $M_{1}=\{\epsilon\} ; \subseteq$ is set inclusion; and $S y\left(M_{0}, M_{1}, \ldots, M_{n}\right)$ is true in $W S 2 S y_{0}$ iff $M_{0}$ is some singleton set $\left\{m_{0}\right\}$ and $S y\left(m_{0}, M_{1}, \ldots, M_{n}\right)$ is true in WS2Sy. We can now translate in a straightforward way any formula $\Phi\left(x_{1}, \ldots, x_{n}, Y_{1}, \ldots, Y_{m}\right)$ of WS2Sy into a formula $\Phi_{0}\left(X_{1}, \ldots, X_{n}, Y_{1}, \ldots, Y_{m}\right)$ of $W S 2 S y_{0}$ such that 


$$
w_{1}, \ldots, w_{n}, M_{1}, \ldots, M_{m} \models \Phi \text { iff }\left\{w_{1}\right\}, \ldots,\left\{w_{n}\right\}, M_{1}, \ldots, M_{m} \models \Phi_{0}
$$

and any formula $\Phi\left(X_{1}, \ldots, X_{n}, Y_{1}, \ldots, Y_{m}\right)$ of $W S 2 S y_{0}$ (for an appropriate enumeration of the variables) into a formula $\Phi_{1}\left(x_{1}, \ldots, x_{n}, Y_{1}, \ldots, Y_{m}\right)$ of WS2Sy such that

$M_{1}, \ldots, M_{n}, N_{1}, \ldots, N_{m} \models \Phi$ iff exists $m_{i}$ such that $M_{i}=\left\{m_{i}\right\}$ for all $i$ and

$$
m_{1}, \ldots, m_{n}, N_{1}, \ldots, N_{m} \models \Phi_{1}
$$

We say that a set $S$ of $n$-tuples of sets of words over $\{1,2\}$ is definable in WS2Sy (resp. restricted WS2Sy or uniform WS2Sy) if there is WS2Sy-formula (resp. restricted WS2Sy-formula or uniform WS2Sy-formula) $\Phi\left(X_{1}, \ldots, X_{n}\right)$ such that

$$
\left(M_{1}, \ldots, M_{n}\right) \in S \text { iff } M_{1}, \ldots, M_{n}=\Phi
$$

According to the translation between WS2Sy and WS2Sy $y_{0}$ that we have mentioned above it does not matter whether we take WS2Sy or WS2Sy $y_{0}$ to define the notion of definability.

\section{Recognizability and Definability}

Before we can formulate the correspondence between definability in (subclasses of) WS2Sy and recognizability by (subclasses of) TACT we need translations of tuples of finite sets of words into trees, and vice versa.

Definition 7. The tree representation of a finite set $W \subset\{1,2\}^{*}$ is the tree $\bar{W} \in T\left(\{0,1\}^{(1)}\right)$ defined by

$$
\bar{W}= \begin{cases}\perp & \text { if } W=\emptyset \\ 0\left(\overline{1^{-1} W}, \overline{2^{-1} W}\right) & \text { if } W \neq \emptyset \text { and } \epsilon \notin W \\ 1\left(\overline{1^{-1} W}, \overline{2^{-1} W}\right) & \text { if } W \neq \emptyset \text { and } \epsilon \in W\end{cases}
$$

where $1^{-1} W=\{w \mid 1 w \in W\}$ and $2^{-1} W=\{w \mid 2 w \in W\}$.

The tree representation $\overline{\left(W_{1}, \ldots, W_{n}\right)}$ of an $n$-tuple $\left(W_{1}, \ldots, W_{n}\right)$ of finite subsets of $\{1,2\}^{*}$ is the tree $t \in T\left(\{0,1\}^{(n)}\right)$ with $t^{\langle i\rangle}=\bar{W}_{i}$ for all $i$.

The tree representation $\bar{S}$ of a set of tuples of finite subsets of $\{1,2\}^{*}$ is $\left\{\overline{\left(W_{1}, \ldots, W_{n}\right)} \mid\left(W_{1}, \ldots, W_{n}\right) \in S\right\}$.

Note that the tree representation of a tuple of sets is uniquely defined since $(\perp \ldots \perp)$ is a constant.

Example 2. The tree representation of the set $\{\epsilon, 2\}$ is the right tree of Figure1,

The tree representation of the triple of sets $(\{\epsilon, 1\},\{1\},\{\epsilon, 2\})$ is the left tree of Figure 1 
Theorem 2. Let $S$ be a set of $n$-tuples of words.

1. If $S$ is definable in restricted WS2Sy then $\bar{S}$ is TACT-recognizable.

2. If $S$ is definable in uniform WS2Sy then $\bar{S}$ is TAT-recognizable.

3. If $S$ is definable in WS2S then $\bar{S}$ is TA-recognizable ([10]).

The proof of the first two items of this theorem is subject of Section 7 , the last item is due to 10 .

Definition 8. The tuple representation $\langle\langle t\rangle\rangle$ of a tree $t \in T\left(\Gamma^{(n)}\right)$ for finite $\Gamma$, say $\Gamma=\left\{c_{1}, \ldots, c_{m}\right\}$, is the $n(m+1)$-tuple of finite subsets of $\{1,2\}^{*}$

$$
\left(M_{0}^{1}, M_{1}^{1}, \ldots, M_{m}^{1}, \ldots, M_{0}^{n}, M_{1}^{n}, \ldots, M_{m}^{n}\right)
$$

defined by

$$
\begin{aligned}
& M_{0}^{i}=\left\{w \mid \text { the } i \text {-th component of the root of }\left.t\right|_{w} \text { is } \perp\right\} \\
& M_{j}^{i}=\left\{w \mid \text { the } i \text {-th component of the root of }\left.t\right|_{w} \text { is } c_{j}\right\}
\end{aligned}
$$

The tuple representation $\langle\langle S\rangle\rangle$ of a set of trees in $T\left(\Gamma^{(n)}\right)$ is $\left.\{\langle t\rangle\rangle \mid t \in S\right\}$.

Example 3. The tuple representation of the middle tree of Figure 1 is

$$
(\{11,12,2\},\{\},\{\epsilon, 1\},\{11,12,2\},\{\epsilon\},\{1\})
$$

Theorem 3. Let $S \subseteq T\left(\Gamma^{(n)}\right)$.

1. If $S$ is TACT-recognizable then $\langle\langle S\rangle\rangle$ is definable in restricted WS2Sy.

2. If $S$ is TAT-recognizable then $\langle\langle S\rangle\rangle$ is definable in uniform WS2Sy.

3. If $S$ is TA-recognizable then $\langle\langle S\rangle\rangle$ is definable in WS2S (【10]).

The proof of the first two items of this theorem is subject of Section 8 , the last item is due to [10].

\section{Translating Formulae to Automata}

In this section we prove Theorem 2 by translating a restricted $W S 2 S y_{0}$-formula $\Phi\left(X_{1}, \ldots, X_{n}\right)$ into a TACT-automaton $A_{\Phi}$ over $\{0,1\}^{(n)}$ such that $A_{\Phi}$ is a TAT when $\Phi$ is uniform, and that $A_{\Phi}$ is a classical tree automaton when $\Phi$ is a $W S 2 S$ formula.

The first step is to construct automata for the atomic formulae of $W S 2 S y_{0}$. Automata for the atomic formulae $X_{1}=X_{2} .0, X_{1}=X_{2} .1, X_{1}=\epsilon$ and $X_{1} \subseteq X_{2}$ can be found in the literature (for instance [4]). It remains to give an automaton for the synchronization predicates: 
The automaton $A_{n}$ is defined as $\left(\{0,1\}, n,\left\{q_{0}, q_{1}\right\},\left\{q_{1}\right\}, \Delta\right)$ where $\Delta$ contains the rules

$$
\begin{aligned}
& \begin{array}{ccc}
(\perp \ldots \perp) & \rightarrow & q_{0}
\end{array} \\
& \left(\perp_{-} \ldots{ }_{-}\right)\left(q_{0}, q_{0}\right) \quad \rightarrow \quad q_{0} \\
& \left(0_{-} \ldots \text { - }_{-}\right)\left(q_{0}, q_{0}\right) \quad \rightarrow \quad q_{0} \\
& \left(1_{-} \ldots \ldots_{-}\right)\left(q_{0}, q_{0}\right) \rightarrow^{1.2=2.2 \wedge \ldots \wedge 1 . n=2 . n} q_{1} \\
& \left(0_{-} \ldots \text {. }_{-}\right)\left(q_{0}, q_{1}\right) \quad \rightarrow \quad q_{1} \\
& \left(0_{-} \ldots_{-}\right)\left(q_{1}, q_{0}\right) \quad \rightarrow \quad q_{1}
\end{aligned}
$$

Proposition 2. Let $S^{n}$ be the set of all $n$-tuples of sets of words such that $S \models S y\left(X_{1}, X_{2}, \ldots, X_{n}\right)$. The language recognized by $A_{n}$ is $\overline{S_{n}}$.

In the case of the equivalence between WS2S and classical tree automata the translation of an arbitrary formula is obtained once the closure of the class of recognizable sets by the boolean operation, cylindrification and projection is established. In case of WS2Sy and TACT we need, however, a more specific property of TACT-recognizable sets than just closure by certain operations: The closure must not affect the set of components on which the automaton performs tests. This invariant of the closure operations is needed because in the closure by projection of the $i$-th component we need as a hypothesis that the automaton does not test on the $i$-th component. An even stronger invariant will be needed to prove that uniform WS2Sy translates to TAT automata.

Proposition 3. If $L$ is recognizable by a TACT $A$ that tests only on the set of components $I$ then there exists a complete and deterministic TACT that tests only on $I$ and that recognizes $L$.

Proof: Completion is achieved by adding a sink state. The determinisation is the same as in [4]. Since all rules in the determined automaton have tests that are boolean combinations of tests in the original automaton the set of tested components is not touched by this operation.

It should be remarked that the determinisation of a tree automaton with tests requires the set of constraints to be closed under negation (see 44).

Proposition 4. If $L$ is recognized by a TACT $A$ that tests only on the set of components $I$ then there exists a TACT that tests only on $I$ and that recognizes $T\left(\Gamma^{(n)}\right)-L$.

If $L_{1}, L_{2}$ are recognized by TACTs $A_{1}, A_{2}$ that test only on the set of components $I$ then there exists a TACT that tests only on $I$ and that recognizes $L_{1} \cup L_{2}$, resp. $L_{1} \cap L_{2}$.

Proof: This is now straightforward (again, see [4]). We just remark that the closure of the language of constraints by conjunction is used in case of the intersection. 
Definition 9. Let $L$ be a set of $\Gamma^{(n)}$-trees. For $1 \leq i \leq n$ the $i$-th projection of $L$ is

$$
\exists i: L=\left\{t^{\langle i\rangle} \mid t \in L\right\}
$$

For $1 \leq i \leq n+1$, the $i+1$-th cylindrification of $L$ is

$$
\uparrow i: L=\left\{t \in T\left(\Gamma^{(n+1)}\right) \mid t^{\langle i\rangle} \in L\right\}
$$

Proposition 5. If $L$ is recognizable by a TACT $A$ that tests only on the set of components $I$ then there exists a TACT that recognizes $\uparrow i: L$ and tests only on $\{j \mid j<i\} \cup\{j+1 \mid j>i\}$.

Proof: Let $L$ be recognized by the TACT $\left(\Gamma, n, Q, Q_{f}, \Delta\right)$. To ease notation we show the construction for the case $i=n+1$. It is straightforward to show that $\uparrow n+1: L$ is recognized by the TACT $\left(\Gamma, n+1, Q, Q_{f}, \Delta^{\prime}\right)$ where

$$
\begin{aligned}
\Delta^{\prime} & =\{(\perp \cdots \perp \perp) \rightarrow q \mid(\perp \cdots \perp) \rightarrow q \in \Delta\} \\
& \cup\left\{(\bar{f} f)\left(q_{1}, q_{2}\right) \rightarrow^{c} q \mid \bar{f}\left(q_{1}, q_{2}\right) \rightarrow^{c} q \in \Delta, f \neq \perp, \bar{f} \neq \bar{\perp}\right\} \\
& \cup\left\{(\bar{\perp} f)\left(q_{1}, q_{2}\right) \rightarrow q \mid(\perp \cdots \perp) \rightarrow q \in \Delta, f \neq \perp\right\}
\end{aligned}
$$

Proposition 6. Let $L \subseteq T\left(\Gamma^{(n)}\right)$ be recognized by a TACT A that tests only on the set of components $I$ and $i \notin I$. There exists a TACT that recognizes $\exists i: L$ and tests only on $\{j \mid j<i\} \cup\{j-1 \mid j>i\}$.

Proof: Let $L$ be recognized by the TACT $A=\left(\Gamma, n+1, Q, Q_{f}, \Delta\right)$. To ease notation we show the construction for the case $i=n+1$.

We can assume w.l.o.g that $A$ is reduced, that is that there is for every $q \in Q$ a term $t \in T\left(\Gamma^{(n)}\right)$ such that $t \rightarrow{ }_{A}^{*} q$ (this can simply be obtained by dropping all states that recognize the empty set). We construct an automaton for $\exists n: L$ as $A=\left(\Gamma, n, Q, Q_{f}, \Delta^{\prime}\right)$ where

$$
\begin{aligned}
\Delta^{\prime} & =\{(\perp \cdots \perp) \rightarrow q \mid(\perp \cdots \perp \perp) \rightarrow q \in \Delta\} \\
& \cup\left\{(\perp \cdots \perp) \rightarrow q \mid(\perp \cdots \perp f)\left(q_{1}, q_{2}\right) \rightarrow q \in \Delta, f \neq \perp\right\} \\
& \cup\left\{\bar{f}\left(q_{1}, q_{2}\right) \rightarrow{ }^{c} q \mid \bar{f} f\left(q_{1}, q_{2}\right) \rightarrow^{c} q, \bar{f} \neq \bar{\perp}\right\}
\end{aligned}
$$

The proof that $A^{\prime}$ accepts $\exists n+1: L$ is exactly as in the case of classical tree automata (see, e.g., [4]). The restriction that the automaton $A$ does not use a test on the $n+1$-th component ensures that the constraints pose no problem here.

Proof of Theorem 2: Let a restricted formula $\Phi$ be given. We can assume without loss of generality that every bound variable of $\Phi$ has exactly one binding quantifier and that no variable of $\Phi$ has both bound and free occurrences in $\Phi$. We construct inductively an automaton for all subformulae of $\Phi$. 
We get an automaton for atomic formulae as in the case of classic tree automata, resp. by Proposition2. Then we cylindrify to have components according to all free and bound variables of $\Phi$. This yields, by Proposition 5 an automaton which does not test on components corresponding to bound variables. In case of composite formulae we conclude by Propositions 4 and 6 .

If the formula $\Phi$ is uniform then the automata corresponding to the atomic formulae have (after cylindrification) only tests True, $1.1=2.1 \wedge \ldots \wedge 1 . n=$ $2 . n$ and $1.1 \neq 2.1 \vee \ldots \vee 1 . n \neq 2 . n$, where we assume for simplicity that the components corresponding to the global variables are $1, \ldots, n$. Since all closure operations on automata construct tests that are boolean combinations of tests of the input automata, the final automaton too has only tests of this form. Since the final automaton works on the components $1, \ldots, n$ it is indeed a TAT.

\section{Translating Automata to Formulae}

In this section we prove Theorem 3 by translating a TACT-automaton $A=$ $\left(\Gamma, n, Q, Q_{f}, \Delta\right)$, where $\Gamma$ has cardinality $m$, into a formula $\Phi$ with $n(m+1)$ free variables.

Let $\Gamma=\left\{c_{1}, \ldots, c_{m}\right\}$, we will use in addition the convention $c_{0}=\perp$. Let $Q=$ $\left\{q_{1}, \ldots, q_{q}\right\}$. We can assume w.l.o.g. that all constraints of the productions of $A$ are conjunctions of equations and inequations since a boolean combination of constraints can be brought into an equivalent disjunctive normal form and a rule $f\left(q_{1}, q_{2}\right) \rightarrow^{c_{1} \vee c_{2}} q$ can be split into two rules $f\left(q_{1}, q_{2}\right) \rightarrow^{c_{1}} q$ and $f\left(q_{1}, q_{2}\right) \rightarrow^{c_{2}} q$.

First we define some auxiliary formulas: The formula partition $\left(Y, Y_{1}, \ldots, Y_{k}\right)$ states that $\left(Y_{1}, \ldots, Y_{k}\right)$ is a partition of $Y$ :

$$
\operatorname{partition}\left(Y, Y_{1}, \ldots, Y_{k}\right)=Y=Y_{1} \cup \ldots \cup Y_{k} \wedge \bigwedge_{i \neq j} Y_{i} \cap Y_{j}=\emptyset
$$

The formula $\operatorname{term}\left(X, X_{1}, \ldots, X_{n(m+1)}\right)$ states that, for some tree $t \in T\left(\Gamma^{(n)}\right)$, $X$ is the set of occurrence of $t$ and $\left(X_{1}, \ldots, X_{n(m+1)}\right)=\langle\langle t\rangle\rangle$ :

$$
\begin{aligned}
\operatorname{term}\left(X, X_{1}, \ldots, X_{n(m+1)}\right)= & \bigwedge_{i=0 \ldots n-1} \operatorname{partition}\left(X, X_{i(m+1)+1}, \ldots, X_{i(m+1)+m+1}\right) \\
& \wedge \forall x, y(x \leq y \wedge y \in X \rightarrow x \in X) \\
& \wedge \forall x\left(x \in X_{0}^{1} \cap \ldots \cap X_{0}^{n} \rightarrow(x 0 \notin X \wedge x 1 \notin X)\right) \\
& \wedge \forall x\left(x \notin X_{0}^{1} \cap \ldots \cap X_{0}^{n} \rightarrow(x 0 \in X \wedge x 1 \in X)\right)
\end{aligned}
$$

The first clause of the formula term says that each component of every occurrence is marked with exactly one symbol and that $X$ is the set of occurrences, the second clause says that the set of occurrences is prefix-closed, the third clause says that any occurrence marked $(\perp \ldots \perp)$ is a leaf and the forth clause that every occurrence not marked with $(\perp \ldots \perp)$ has two subtrees. Finally, the formula is 


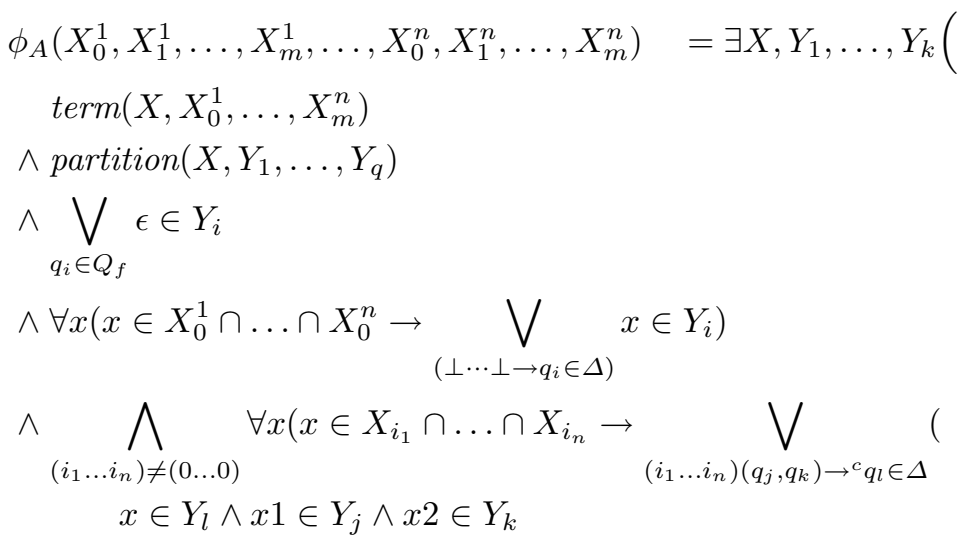

$$
\left.\left.\left.\wedge \bigwedge_{1 . i=2 . i \in c} S y\left(x, X_{0}^{i}, \ldots, X_{m}^{i}\right) \wedge \bigwedge_{1 . i \neq 2 . i \in c} \neg S y\left(x, X_{0}^{i}, \ldots, X_{m}^{i}\right)\right)\right)\right)
$$

In this formula, the first conjunct says that the free variables are the encoding of a $\Gamma^{(n)}$-tree with set of occurrences $X$. The second conjunct says that every occurrence of the tree is assigned a state. The third conjunct expresses that the root of the tree is assigned an accepting state, and the last two conjuncts express that the assignment is according to the transitions rules of the automaton: The forth conjunct covers the case of the constant $(\perp \ldots \perp)$ and the complex last conjunct the case of a binary function symbol.

Note that the formula $\phi_{A}$ is restricted. If the automaton is in fact a TAT automaton then the formula $\phi_{A}$ is equivalent to a uniform WS2Sy-formula since

$$
S y\left(x, X_{0}^{i}, \ldots, X_{m}^{i}\right) \wedge S y\left(x, X_{0}^{j}, \ldots, X_{m}^{j}\right)
$$

is equivalent to the formula

$$
\operatorname{Sy}\left(x, X_{0}^{i}, \ldots, X_{m}^{i}, X_{0}^{j}, \ldots, X_{m}^{j}\right)
$$

Finally, if the automaton $A$ is a classical tree automaton then $\phi_{A}$ contains no synchronization predicate and is hence a WS2S-formula.

\section{Other Classes of Automata with Tests}

So far we have only considered the class of automata with tests between brothers introduced in 11. We can easily extend the results of this paper to classes of automata with "deep" tests that can perform tests like $11 . i=222 . i$, where a tree $t$ satisfies this test if $\left(\left.t\right|_{11}\right)^{\langle i\rangle}=\left(\left.t\right|_{222}\right)^{\langle i\rangle}$ [5]. This generalization is easily achieved by defining a generalization of WS2Sy with stronger synchronization predicates. These classes of automata are, however, of limited interest since already the class of automata with tests between cousin positions (that is $p . i=q . i$ where $p$ and $q$ have length 2) has an undecidable emptiness problem [9].

There is a very interesting class of tree automata with tests that has a decidable emptiness problem: the class of reduction automata [3] and generalized 
reduction automata [2]. These automata allow "deep" (in the above sense) tests but come with a syntactic restriction that ensures that on every branch of a tree only a bounded number of equality tests can be performed. Hence, the undecidability proof of this paper does not directly apply to a generalization of these automata to component-wise tests since we used the fact that we can perform an unbounded number of equality tests on the left spine of the tree (see Section 4). Hence, it is still possible that the results known for (generalized) reduction automata can be lifted to component-wise tests, and that a corresponding logic with a restriction on the set of admissible formulae can be found.

\section{References}

1. B. Bogaert and S. Tison. Equality and disequality constraints on brother terms in tree automata. In P. Enjalbert, A. Finkel, and K. W. Wagner, editors, 9th Annual Symposium on Theoretical Aspects of Computer Science, volume 577 of Lecture Notes in Computer Science, pages 161-171, Paris, France, 1992. Springer-Verlag.

2. A.-C. Caron, H. Comon, J.-L. Coquidé, M. Dauchet, and F. Jacquemard. Pumping, cleaning and symbolic constraints solving. In Proc. Int. Conference on Algorithms, Languages and Programming, pages 436-449, 1994.

3. A.-C. Caron, J.-L. Coquidé, and M. Dauchet. Encompassment properties and automata with constraints. In C. Kirchner, editor, 5th International Conference on Rewriting Techniques and Applications, volume 690 of Lecture Notes in Computer Science, pages 328-342, Montreal, Canada, June 1993. Springer-Verlag.

4. H. Comon, M. Dauchet, R. Gilleron, F. Jacquemard, D. Lugiez, S. Tison, and M. Tommasi. Tree automata. Techniques and Applications, Apr. 1999. Available at http://www .grappa.univ-lille3.fr/tata/.

5. J. Mongy. Transformations de noyaux reconnaissables d'arbres. Forêts RATEG. $\mathrm{PhD}$ thesis, Laboratoire d'Informatique Fondamentale de Lille, Université Lille 1, Villeneuve d'Asq, France, 1981. In French.

6. E. L. Post. A variant of a recursively unsolvable problem. Bulletin of the AMS, 52:264-268, 1946.

7. M. Rabin. Decidability of second-order theories and automata on infinite trees. Trans. Amer. Math. Soc., 141:1-35, 1969.

8. F. Seynhaeve. Automates, Réécriture et Contraintes : Résultats de Décidabilité et d'Indécidabilité. PhD thesis, Université de Lille 1, Villeneuve d'Ascq, France, May 1999. In French.

9. F. Seynhaeve, M. Tommasi, and R. Treinen. Grid structures and undecidable constraint theories. In M. Bidoit and M. Dauchet, editors, Theory and Practice of Software Development, volume 1214 of Lecture Notes in Computer Science, pages 357-368, Lille, France, Apr. 1997. Springer-Verlag. Extended version to appear in Theoretical Computer Science.

10. J. Thatcher and J. Wright. Generalized finite automata with an application to a decision problem of second-order logic. Math. Systems Theory, 2:57-82, 1968. 